\section{RAPAMYCIN HITS THE TARGET}

Charles Sawyers and colleagues have conducted a small early-stage clinical study to assess target modulation by the small-molecule inhibitor rapamycin. Rapamycin is approved to treat renal cancer and is in clinical trials to treat various other cancers, but the molecular determinants of drug response are unknown.

Preclinical data show that loss of the tumour suppressor PTEN sensitizes tumours to the inhibition of mammalian target of rapamycin (mTOR). Sawyers and colleagues conducted a phase I trial of rapamycin in patients with recurrent glioblastoma whose tumours lacked expression of PTEN and who had not had previous mTOR inhibitor therapy. Glioblastoma was chosen for this proof-of-concept study for several reasons. First, PTEN inactivation occurs in about $40 \%$ of patients. Second, salvage surgical resection is often done following relapse after standard therapy (typically, surgical resection plus adjuvant therapy). This allows collection of tumour tissue to assess the molecular effects of treatment given preoperatively. Third, as glioblastomas are highly proliferative they are a good model to assess cytostatic activity - a characteristic of rapamycin treatment observed preclinically.

The 15 patients in the trial received neoadjuvant rapamycin before salvage surgical resection and, after recovery from surgery, patients continued on rapamycin until tumour progression was seen. Tumour samples were taken during both resections from these 15 patients and from nine controls: patients who did not receive rapamycin.

Tumour cell proliferation was reduced in half of the rapamycin-treated patients, but no change in proliferation was seen in the controls. The magnitude of proliferation reduction correlated with mTOR inhibition but not with intratumoural concentrations of rapamycin. Phosphorylation of the S6 ribosomal protein, a downstream effector of mTOR action, was reduced in the salvage surgery samples compared with the initial surgery samples in the patients treated with rapamycin, but no change was seen between the two matched samples collected from the control patients. Interestingly, the magnitude of 56 phosphorylation inhibition did not correlate with intratumoural rapamycin concentrations. Sensitivity to the resistant tumour cells was restored in ex vivo culture, indicating that it is not a cell-intrinsic mechanism but delivery of rapamycin to tumour cells that is impaired in some patients.

As mTOR inhibitors can inhibit a negative feedback loop that regulates activation of AKT, which is upstream in the mTOR pathway, the authors also assessed AKT activity in the matched surgical samples. An increase in AKT activity was observed in the salvage surgery samples of $50 \%$ of the patients. Importantly, this induction of AKT was associated with a shorter time to progression.

This trial shows the importance of investigating drug delivery to tumour cells and target modulation in patients to guide future clinical development of targeted agents. Further study of rapamycin in PTEN-deficient glioblastoma is warranted.

ORIGINAL RESEARCH PAPER Cloughsey, T. F. et al. Antitumor activity of rapamycin in a phase I trial for patients with recurrent PTEN-deficient glioblastoma. PLoS Med. 5, e8 (2008) 